\title{
Local strategic control of information in visual word recognition
}

\author{
HYEWON KANG \\ Sung Kyun Kwan University, Seoul, South Korea \\ and \\ GREG B. SIMPSON \\ University of Kansas, Lawrence, Kansas
}

\begin{abstract}
In the present studies, we examined strategic flexibility in the use of lexical and sublexical information in Korean word recognition. Korean readers show a large frequency effect for words printed in the alphabetic Hangul script only if these words are embedded in a list consisting largely of logographic (Hanza) words. In the first experiment, high- and low- frequency Hangul words were preceded by Hangul or Hanza words. Frequency effects were eliminated when a Hangul target word was preceded by two Hangul words, even when the overall proportion of Hanza words in the list was large. In the second experiment, one group saw each stimulus preceded by a cue indicating which script would be seen on that trial. Frequency effects for Hangul targets were absent under this condition, but were present when subjects were not so cued. These results indicate that Korean readers are able to control their use of lexical and sublexical information over a small number of stimuli or even trial by trial.
\end{abstract}

In the past two decades, a large number of studies have addressed how a printed word or letter string is translated into a phonological representation when people read aloud (see Frost, 1998, for a thorough review). This issue is frequently discussed in terms of the relationships among sources of information (e.g., orthographic, phonological, and semantic) relevant to the identity of a word. Recently, some researchers have suggested that readers have some control over the degree to which these various relationships are emphasized during word recognition.

Monsell, Patterson, Graham, Hughes, and Milroy (1992) examined the naming of words and nonwords either in pure lists of words or nonwords or in lists mixing the two. Both for high-frequency irregular words and nonwords, naming was faster in pure than in mixed lists. In addition, irregular words showed more regularization errors when embedded in lists including nonwords than in pure word lists. These results suggest that readers change their wordrecognition strategies to conform to the characteristics of the stimuli they encounter.

Results such as these have most often been interpreted in terms of the dual-route conception of reading (e.g., Coltheart, Curtis, Atkins, \& Haller, 1993), according to which there are two pathways for pronouncing printed stimuli. In the lexical route, there is a direct mapping be-

The authors are grateful to Yongwon Suh for his help in conducting the research, and we thank George Kellas, Jason Zevin, and an anonymous reviewer for their comments on an earlier draft. Correspondence concerning the article may be addressed to G. Simpson, Department of Psychology, University of Kansas, 1415 Jayhawk Blvd., Lawrence, KS 66045-7556 (e-mail: gsimpson@ku.edu). tween a whole-word orthographic pattern and the word's pronunciation stored in the lexicon. The sublexical route generates an assembled representation that involves a mapping between subword orthographic units (graphemes) and corresponding phonological units (phonemes) via the application of grapheme-phoneme correspondence (GPC) rules. The latter route allows the pronunciation of unfamiliar words or nonwords that are not represented in the lexicon. The former is necessary for the pronunciation of irregular words that do not follow GPC conventions. In these terms, the presentation of pure or mixed blocks of stimuli (as in Monsell et al., 1992) influences the relative utility of each of these routes. The introduction of nonwords biases the reader to emphasize sublexical GPC information, thus leading to more frequent mispronunciations of irregular words.

In contrast, parallel distributed processing (PDP) models (e.g., Plaut, McClelland, Seidenberg, \& Patterson, 1996) do not recognize a distinction between a lexicon and a rule-governed assembly process. Rather, recognition of a letter string involves a pattern of activation over processing units associated with that string's orthographic, phonological, and semantic characteristics. Pronunciation of words (regular or irregular) and nonwords is always achieved by the activation of a phonological pattern on the basis of the input orthographic pattern. That is, these models do not acknowledge different processing operations for different types of stimuli. However, characteristics of tasks and stimuli may affect the "division of labor" among the sources of information (Seidenberg, 1992). Whereas word naming will emphasize the connections between orthography and phonology (which benefit from 
regular orthography-phonology relationships), a semantic categorization task may emphasize the orthographicsemantic connections more (which do not so benefit). Likewise, the proportions of words and nonwords in a list may affect the degree to which semantic information is used in the translation from print to pronunciation.

Much of the research investigating flexible control over information used in word naming has been conducted in languages other than English, particularly those represented by "shallow" orthographies (such as Italian, SerboCroatian, and Korean), in which the mapping between graphemes and phonemes is very consistent. Readers of a shallow orthography may rely more heavily on sublexical information, because it is a more reliable source of the pronunciation code than it is in a deeper orthography (such as in English), in which the mapping from print to sound is much less consistent. In such studies, researchers have used semantic priming or word frequency effects as "markers" of the use of lexical or sublexical information. The focus on these factors is based on the dual-route theory's assumption that such effects originate in the lexicon: High-frequency words are found in the lexicon more rapidly than are low-frequency words, and spreading activation within the lexicon allows for faster recognition of words preceded by a semantically related prime. On the other hand, the GPC rules that drive the sublexical path operate on the basis of subword units, so they should be less affected by word-level factors. Alternatively, a PDP account (Seidenberg, 1992) would place responsibility for these effects on the relative strength of orthographicphonological connections possible in a shallow orthography. That is, because the system is exposed only to consistent mappings between orthographic and phonological units, the connections among these units are very strong and, in a sense, overshadow the effects of other contributions to word naming. On either account, because of the greater consistency that shallow orthographies show between print and pronunciation, readers of such languages should show smaller effects of word-level characteristics than seen in deeper orthographic systems. This prediction is confirmed in the finding that both frequency and priming effects are smaller in shallow-orthography languages, such as Italian (Tabossi \& Laghi, 1992), SerboCroatian (Frost, Katz, \& Bentin, 1987), and Korean (Kang \& Simpson, 1996; Simpson \& Kang, 1994).

The present research extends that of Simpson and Kang (1994), conducted in Korean. The Korean writing system possesses several characteristics that make it highly suitable for addressing questions of lexical and sublexical processing. The dominant script, called Hangul, is an alphabet that is very consistent in its mapping between graphemes and phonemes. However, Korean also continues to use a substantial number of Chinese characters. This script, called Hanza, is logographic and orthographically very deep. Hanza words are interchangeable with Hangul words, in that words written in Hanza can be, and often are, written in Hangul as well. There are, however, a large number of words that can be written only in Hangul (see Table 1 for examples of Hangul and Hanza words).
Table 1

Examples of Korean Words Written in Both Hangul and Hanza

\begin{tabular}{lccc}
\hline English word & Hangul & Hanza & Pronunciation \\
\hline Classroom & 교실 & 教室 & $/$ gyo-sil/ \\
Nation & 국가 & 國家 & $/$ guk-ga/ \\
Literature & 문학 & 文學 & $/$ mun-hak/ \\
\hline
\end{tabular}

Previous research suggests differences in the way in which Hangul and Hanza words are processed. Phonological information plays a more prominent role in the semantic processing of Hangul than in that of Hanza (Cho \& Chen, 1999). In addition, Hangul processing is very sensitive to the presence of Hanza, whereas Hanza word recognition is unaffected by being mixed with Hangul (Simpson \& Kang, 1994, Experiment 1).

Simpson and Kang (1994, Experiment 2) had native Korean speakers name high- and low-frequency Hangul words in the presence either of additional Hangul words, Hanza words, or pronounceable Hangul nonwords. If readers have control over the type of information emphasized in processing alphabetic Hangul words, then, in the presence of a large number of Hanza words, which can only be processed lexically, Hangul words should be processed in this way also. When only Hangul is seen, however, sublexical information can reliably generate a correct pronunciation and may be used more. We found that when Hanza dominated the stimulus list, Hangul words showed a large frequency effect. When only Hangul words or nonwords were presented, however, the frequency effect was diminished substantially. These results were interpreted to mean that Korean readers are able to control processing routes in alphabetic word recognition, using sublexical information when only the shallow Hangul alphabet is seen, but relying on lexical information in the presence of a large number of logographic Hanza words.

The implicit assumption in most studies examining strategic processing in visual word recognition (e.g., McQuade, 1981; Monsell et al., 1992) is that subjects adopt a strategy during the early trials of the experiment as they discern the distribution of stimulus types across those trials. This assumption may be made more explicit in the deliberate biasing of practice trials intended specifically to induce a particular strategy (e.g., Monsell et al., 1992). The development of a strategy over the course of an experiment should be discernible in the data. Specifically, we would expect that if we compared the early and late trials of an experiment, we should see more evidence of the strategy in the late portion. However, the results reported by Simpson and Kang (1994) did not show this pattern, in that the frequency effect did not change across the course of the experiment. We hypothesize that the strategic control that subjects had over processing in that study was sensitive to the distribution of the immediately preceding trial or trials. In the Hanza filler condition, $80 \%$ of the stimuli were printed in Hanza. Because words were presented in a random order, a Hangul target usually followed a Hanza word. If the subject is influenced by the type of word presented immediately prior to the current 
target, this would lead to a pattern indistinguishable from a list-wide strategy, but it would not lead to a pattern of change across the experiment. As there was no evidence of such a change in Simpson and Kang's results, we hypothesize that the control of processing was operating over a smaller window of stimuli. A pattern consistent with this hypothesis has been found recently by Taylor and Lupker (2001). In that research, naming time both for words and nonwords (in lists mixing these stimuli) were affected by the lexical status of the preceding stimulus.

\section{EXPERIMENT 1}

The purpose of the first experiment was to test whether strategic control in processing alphabetic Korean operates locally, over a small number of sequentially presented stimuli, or globally, over an entire experimental list. Subjects named high- and low-frequency alphabetic Hangul words in lists consisting of $80 \%$ Hangul or $80 \%$ Hanza words. Stimulus order within the list was controlled so that each critical Hangul target was preceded immediately by one Hangul, two Hangul, one Hanza, or two Hanza word(s). In this way, we were able to manipulate independently the global context (the distribution of Hangul and Hanza scripts across the entire experimental list) and the local context (the script of the immediately preceding one or two words). If processing is dictated by a global judgment about which path will lead to optimal performance, we expect to see large frequency effects when $80 \%$ of the stimuli are Hanza. The frequency effect should be smaller when the majority of stimuli are printed in Hangul. On the other hand, if processing is driven by the local distribution of a small number of preceding stimuli, we expect frequency effects for Hangul words preceded by Hanza, but not for those preceded by other Hangul words, regardless of the composition of the list as a whole.

\section{Method}

Subjects. Forty-one native speakers of Korean initially participated in this experiment. All were students at Sung Kyun Kwan University in Seoul, South Korea, and received course credit for their participation. Nine were eliminated from the original pool because of excessive error rates (>15\%) in naming Hanza words, indicating that their proficiency in reading Hanza (which can vary considerably among Korean readers) was not high enough to assess strategic effects resulting from Hanza reading. The data reported, therefore, are from the 32 subjects who were under the $15 \%$ error criterion. Neither the mean Hanza error proportions nor response latencies for Hanza words differed between those subjects assigned to the $80 \%$ Hangul and the $80 \%$ Hanza conditions (both $t \mathrm{~s}<1$ ).

Stimuli. The critical stimuli were 32 high-frequency and 32 lowfrequency two-syllable Hangul words, taken from Korean wordfrequency norms (Lee et al., 1991). High-frequency words were in the 99th percentile of printed Hangul frequency, and low-frequency words were in the 27th percentile. High- and low-frequency words were matched on initial phoneme and number of phonemic elements per syllable and did not differ either in summed syllable or summed bigram frequency (also available from the Lee et al. norms, both $t \mathrm{~s}<1$ ).

To manipulate the local script context independently of the overall script proportion, a list was formed consisting of a fixed order of word types (i.e., Hangul and Hanza words). This ensured that high- and low-frequency Hangul words were preceded equally often by one Hangul, two Hangul, one Hanza, or two Hanza words. Next, an initial set of filler stimuli was added to serve as the words preceding the critical Hangul words. These fillers consisted of 32 additional Hangul words and 96 Hanza words (fewer Hangul fillers were needed because a Hangul word can be both a critical word itself and also a preceding stimulus for another critical word). Finally, an additional 288 filler words were added to create the final 480 word stimulus lists. For one list, these fillers were Hangul, and, for the other, they were Hanza. These fillers were in fact the same words, selected initially for their Hanza familiarity (all were selected from middle-school textbooks used in the teaching of Hanza) and then transcribed into Hangul. In this way, the final stimulus lists achieved the appropriate proportion of the two scripts (i.e., either $80 \%$ Hangul or $80 \%$ Hanza). These filler stimuli were added so as not to disrupt the relationship between the critical Hangul words and their designated preceding stimuli and also to prevent subjects from being able to predict the script of any single upcoming stimulus. ${ }^{1}$ Four lists were ultimately created for each script condition, to ensure that each critical Hangul word was preceded equally often by one Hangul, two Hangul, one Hanza, or two Hanza words.

Design and Procedure. The design for this experiment was a 2 $\times 2 \times 2 \times 2$ mixed factorial, with overall filler script that dominated the list (Hangul or Hanza) manipulated between subjects, and critical-word frequency (high or low), local context script (Hangul or Hanza), and number of preceding words (one or two words of the context script) as the within-subjects factors. Note that filler script refers to the dominant script in the list as a whole, whereas context script refers to the script of the one or two words preceding a critical Hangul target.

The subjects were tested individually, seated in front of a microphone and the monitor of an IBM-compatible computer running the Micro Experimental Laboratory (MEL) software system (Schneider, 1988) and software for presenting stimuli in Hangul and Hanza. The microphone was connected to the computer via a response box for collecting naming latencies. The experimenter initiated each trial by pressing a key on the response box. After $500 \mathrm{msec}$, a warning signal (a plus sign) was presented at the center of the screen for $500 \mathrm{msec}$. Two-hundred milliseconds following the offset of the plus sign, the word appeared, also centered on the screen. The subjects were instructed to name the word aloud as quickly and as accurately as possible. Presentation of the word activated a timer, which was stopped with the initiation of the naming response into the microphone. The experimenter recorded the accuracy of the response according to six possible outcomes: (1) correct responses, (2) incorrect responses, (3) spoiled responses (e.g., activation by coughs or failure to activate the response key), (4) responses with an excessive pause between the pronunciation of the two syllables, (5) partially incorrect responses (i.e., incorrect pronunciation of one of the two syllables), and (6) failures to respond due to lack of Hanza knowledge. After the experimenter registered the appropriate outcome, the start key was pressed again to begin the next trial.

The subjects first received 15 practice trials. Those assigned to the Hangul filler condition received a random order of 12 Hangul and 3 Hanza words, and the subjects assigned to the Hanza filler condition received a random order of 3 Hangul and 12 Hanza words. They then completed the 480 experimental trials, divided into four blocks of 120 trials, with a short break after each block. The entire experiment took approximately $40 \mathrm{~min}$ for a single subject to complete.

\section{Results and Discussion}

Mean naming latencies for correct responses to highand low-frequency Hangul critical words are shown in Table 2. ${ }^{2}$ Errors (incorrect and partially incorrect responses) were very rare $(0.15 \%$ of critical words), resulting in 14 (of a possible 16) cells with no errors. Conse- 
Table 2

Mean Naming Latencies in Milliseconds for High-Frequency (HF) and Low-Frequency (LF) Targets, and Frequency Effect (FE) as a Function of Filler Script, Context Script, and Number of Context Script Words

\begin{tabular}{llllllll}
\hline & \multicolumn{3}{c}{ Hangul Filler List } & & \multicolumn{3}{c}{ Hanza Filler List } \\
Context and Number & HF & LF & FE & & HF & LF & FE \\
\hline Hangul & & & & & & \\
1 (Hanza-Hangul) & 574 & 603 & 29 & & 597 & 634 & 37 \\
2 (Hangul-Hangul) & 579 & 576 & -3 & 603 & 614 & 11 \\
Hanza & & & & & & \\
1 (Hangul-Hanza) & 583 & 612 & 29 & 614 & 642 & 28 \\
2 (Hanza-Hanza) & 595 & 626 & 31 & 619 & 653 & 34 \\
\hline
\end{tabular}

quently, naming errors are not discussed further. Spoiled responses, which constituted $1.03 \%$ of critical-word responses, were eliminated from the naming latency analysis. Responses with a pause between the two syllables and failures to respond never occurred for critical words.

Naming latencies of Hangul critical words were analyzed in 2 (filler script) $\times 2$ (word frequency) $\times 2$ (local context script) $\times 2$ (number of preceding words of the context script) analyses of variance (ANOVAs), treating subjects $\left(F_{1}\right)$ and targets $\left(F_{2}\right)$ as random factors (Clark, 1973). In the subjects analysis, filler script was the only between-subjects factor, and all other variables were within subjects. In the items analysis, word frequency was the only between-words factor, with all others within words. Unless otherwise noted, all tests assume a minimum level of significance of $p<.05$.

The results showed a significant main effect of filler script by items $\left[F_{2}(1,62)=67.24, M S_{\mathrm{e}}=1,445.55\right]$, though not by subjects $\left[F_{1}(1,30)=2.18, M S_{\mathrm{e}}=23,855.80\right.$, $p>.15]$, with Hangul critical words named $28 \mathrm{msec}$ faster in the Hangul filler than in the Hanza filler condition. No interaction effects involving filler script were significant.

Of primary interest is the significant three-way interaction of local context script, number of preceding words, and word frequency $\left[F_{1}(1,30)=5.00, M S_{\mathrm{e}}=840.84\right.$; $\left.F_{2}(1,62)=5.49, M S_{\mathrm{e}}=1,676.41\right]$. This interaction was examined by analyzing the two-way effects of number of preceding items and frequency separately for the Hangul and Hanza local context script conditions. Number of preceding words interacted with frequency only in the Hangul condition $\left[F_{1}(1,31)=9.85, M S_{\mathrm{e}}=663.43 ; F_{2}(1,126)=\right.$ 9.39, $\left.M S_{\mathrm{e}}=1,568.37\right]$. When a critical target was preceded by one Hangul word, there was still a significant word frequency effect $\left[F_{1}(1,31)=26.91, M S_{\mathrm{e}}=633.96\right.$; $\left.F_{2}(1,126)=11.21, M S_{\mathrm{e}}=3,485.36\right]$, which disappeared when critical words were preceded by two Hangul words (both $F_{\mathrm{S}}<1$ ). When target stimuli were preceded by Hanza fillers, on the other hand, significant word frequency effects were observed regardless of whether there was one preceding Hanza word $\left[F_{1}(1,31)=16.86, M S_{\mathrm{e}}=\right.$ $\left.783.18 ; F_{2}(1,126)=7.99, M S_{\mathrm{e}}=3,276.91\right]$ or two preceding Hanza words $\left[F_{1}(1,31)=17.97, M S_{\mathrm{e}}=944.33\right.$; $\left.F_{2}(1,126)=10.73, M S_{\mathrm{e}}=3,091.16\right]$. In other words, only when critical Hangul words were preceded by two additional Hangul was there no effect of word frequency. Further, this pattern was not affected by the overall proportion of Hangul and Hanza words in the entire list, as the four-way interaction (filler script $\times$ local context script $X$ number of preceding words $X$ word frequency) was not significant (both $F_{\mathrm{S}}<1$ ).

Simpson and Kang (1994) showed that Hangul wordfrequency effects were present only in stimulus lists that contained a large number of logographic Hanza words. The present results indicate, however, that it was not the overall construction of the list that determined performance as much as it was the local distribution of stimuli within the list. Even when the list was dominated by Hanza words, the Hangul word-frequency effect was diminished if the critical targets were preceded by two Hangul words. Conversely, the frequency effect was substantial even in Hangul-dominated lists if critical words were preceded by even a single Hanza word.

The finding of an immediate effect of stimulus type on the processing of upcoming words is consistent with recent similar reports. Taylor and Lupker (2001) showed that both words and nonwords were named more quickly when they followed "fast" stimuli (high-frequency regular words) than when they followed "slow" stimuli (nonwords). In a study examining the processing of Japanese Kanji and Kana scripts, Shafiullah and Monsell (1999) found that the reading of either script was slower following a change from one script to the other than when the script was held constant across trials. To the best of our knowledge, however, the present study is the first that has directly compared such local context to global list context.

In the procedures used by Simpson and Kang (1994; and most other strategy studies as well), the proportion of stimulus types is manipulated within a random ordering of the stimuli. Under these conditions, it is very difficult to distinguish between local and list-wide strategies, because critical stimuli are more likely to be preceded by whatever stimulus type dominates the list. Therefore, even if processing is influenced only by the immediately preceding stimulus, it will appear as if the overall proportion is responsible. By deliberately ordering the stimulus conditions, we have been able to separate these two possible sources of control, and the results indicate an important role for local control.

We do not mean to imply that there are no effects of overall list construction. When two Hangul context words were presented, the frequency effect was larger in the Hanza-filler condition (11 vs. $-3 \mathrm{msec}$ for the Hangulfiller condition). Although the relevant interaction did not approach significance, this pattern suggests a list-wide contribution to strategic effects in naming. In addition, the main effect of filler script shows that Hangul stimuli are named more rapidly in Hangul-dominated than in Hanza-dominated lists (see also Simpson \& Kang, 1994). The latter result is consistent with an alternative account of strategy effects, which will be considered in the General Discussion section. 


\section{EXPERIMENT 2}

The purpose of the second experiment was to examine the possibility of an even more deliberate form of local control and also to test whether there are any circumstances under which sublexical processing can be initiated based only on immediately preceding information. High- and low-frequency Hangul words were embedded in a list dominated by Hanza words. This list construction led to a very large frequency effect for Hangul words in Simpson and Kang (1994). In the present experiment, however, the subjects in one group were cued before each trial as to which script would be used for the word on that trial. If Korean readers are able to exert immediate control over lexical and sublexical processing, the cued group should show smaller Hangul frequency effects than should a group that is not cued. The former group will know when a Hangul word is to appear and may rely more on sublexical information, thus reducing the frequency effect. Because the list is dominated by Hanza words, however, an uncued group should be influenced only by the proportions of stimulus types and show the large frequency effect typical of readers viewing such lists.

\section{Method}

Subjects. Originally, 42 native speakers of Korean (students at Sung Kyun Kwan University) participated. None had participated in Experiment 1. As in Experiment 1, subjects' naming performance on a subset of the Hanza words used in the experiment (the same 96 Hanza words used in Experiment 1) was used to determine Hanza proficiency. Ten of the original 42 subjects were so eliminated, having committed more than $15 \%$ naming errors on Hanza words. The remaining 32 subjects were randomly assigned to the cued or uncued groups. The groups did not differ in Hanza naming latency or accuracy (both $t \mathrm{~s}<1$ ).

Stimuli. Stimuli consisted of 200 two-syllable Korean words. Critical stimuli were 20 high-frequency and 20 low-frequency Hangul words selected from the Korean word frequency norms (Lee et al., 1991). High- and low-frequency words were matched according to initial phoneme and the number of graphemic elements per syllable. In addition, the high- and low-frequency words did not differ in the summed frequency of their two syllables or in their summed bigram frequency (both $t \mathrm{~s}<1$ ).

The remaining 160 stimuli ( $80 \%$ of the stimulus list) were twosyllable Hanza words, selected from among the same population of Hanza words described in Experiment 1. Because of the disproportionate construction of the list (i.e., the large number of Hanza words), a fixed order of stimuli was developed, determined randomly with the restrictions that no more than two Hangul or six Hanza words were presented consecutively

Design and Procedure. The design for this experiment was a $2 \times 2$ mixed factorial, with script information (cued or uncued) manipulated between subjects and Hangul word frequency (high- or low-frequency) as the within-subjects variable. In the cued condition, a color was assigned to each script (green to Hangul and red to Hanza), and the warning fixation stimulus (four asterisks) was presented in one of these colors. The subjects were informed that when the green fixation stimulus was presented, the following word would be printed in Hangul and that a red fixation stimulus would always be followed by a Hanza word. In the uncued condition, all fixation stimuli were printed in white. Prior to the experiment, the subjects assigned to the cued group were presented with 10 trials in which they were asked to identify the color of the stimuli (again, green or asterisks) presented on the computer screen. No subject made any errors on these color-naming trials.

Because it was very important that subjects attend to the fixation stimulus for its entire duration (to ensure that equivalent information was derived from the cue on each trial), a warning signal for the prime was included. Therefore, each trial began with the presentation of a $1000-\mathrm{Hz}$ tone lasting $50 \mathrm{msec}$. Five-hundred milliseconds following the offset of the tone, the fixation stimulus (four asterisks) was presented at the center of the screen for 1,000 msec. For the uncued group, this stimulus was always printed in white; for the cued group, it was green on $20 \%$ of the trials (Hangul trials) and red on $80 \%$ (Hanza trials). Two-hundred milliseconds following the offset of the fixation, the target was presented at the same location, starting the millisecond timer, and remained on the screen until the subject's naming response was made. The subjects were instructed to name the target as quickly and as accurately as possible.

The subjects first received 30 practice trials. The first 10 of these consisted of a random ordering of 5 Hangul and 5 Hanza words to enable subjects in the cued condition to become familiar with the mapping between color and script. The next 20 trials consisted of a random order of 4 Hangul and 16 Hanza words (the proportions represented in the experimental trials). Although the first 10 practice trials were not necessary for subjects in the uncued condition (since they were not to see any colored fixation stimuli), both groups received the full 30 trials. They then completed 200 experimental trials, divided into two blocks of 100 trials each, with a short break between blocks. The entire experiment took approximately $30 \mathrm{~min}$ for a subject to complete.

\section{Results and Discussion}

Mean naming latencies for correct responses to highand low-frequency Hangul words are shown in Table 3. Errors - fully or partially incorrect responses - were very rare $(0.23 \%$ of Hangul words), resulting in no errors in three of the four cells. Consequently, errors were not analyzed and will not be discussed further. Spoiled responses constituted $1.17 \%$ of critical words and were eliminated from the naming latency analysis.

Naming latencies of Hangul words were submitted to 2 (cued vs. uncued) $\times 2$ (high- vs. low-frequency) ANOVAs, treating subjects $\left(F_{1}\right)$ and words $\left(F_{2}\right)$ as random factors. In the subjects analysis, script cuing was the between-subjects factor, and frequency was the withinsubjects factor. In the words analysis, script cuing was a within-words variable, whereas frequency was examined between words.

The interaction of cuing and frequency was significant both by subjects $\left[F_{1}(1,30)=12.30, M S_{\mathrm{e}}=370.59\right]$ and by words $\left[F_{2}(1,38)=13.57, M S_{\mathrm{e}}=388.70\right]$. Simple effects analyses showed a significant word frequency effect in the uncued condition $\left[F_{1}(1,15)=24.01, M S_{\mathrm{e}}=\right.$ $\left.512.25 ; F_{2}(1,38)=11.03, M S_{\mathrm{e}}=1,289.35\right]$, with high-

Table 3

Mean Naming Latencies in Milliseconds for High-Frequency (HF) and Low-Frequency (LF) Targets, and Frequency Effect (FE) as a Function of Cuing Condition

\begin{tabular}{cccc}
\hline & \multicolumn{3}{c}{ Target Frequency } \\
\cline { 2 - 4 } Cuing Condition & HF & LF & FE \\
\hline Cued & 625 & 631 & 6 \\
Uncued & 623 & 662 & 39 \\
\hline
\end{tabular}


frequency Hangul words named $38 \mathrm{msec}$ faster than lowfrequency Hangul words. The 6-msec frequency effect in the cued condition, however, was not significant $\left[F_{1}(1,15)=1.04, M S_{\mathrm{e}}=228.92 ; F_{2}<1\right]$.

The data from the uncued condition replicate those reported by Simpson and Kang (1994), by showing a large word frequency effect for Hangul words presented in the context of a large number of logographic Hanza words. This is expected based on the assumption that Hanza, as it requires lexical processing, biases the reader toward continued use of this path for alphabetic Hangul words as well. This bias may be overcome, however, by advance knowledge of the script to be presented on a given trial. If the subject knows that a particular trial will display a Hangul word, he or she may change processing so as to increase the use of sublexical information in arriving at a pronunciation. These results suggest even more precise control over processing than was suggested by Experiment 1 . In the present case, the subjects were able to adjust processing on a trial-by-trial basis, simply on the basis of prior knowledge of the script in which a word was to be printed.

\section{GENERAL DISCUSSION}

The results extend those of previous research showing that readers are able to emphasize lexical or sublexical information in word naming, depending on characteristics of the stimulus context (Baluch \& Besner, 1991; Besner \& Hildebrandt, 1987; McQuade, 1981; Milota, Widau, McMickell, Juola, \& Simpson, 1997; Monsell et al., 1992; Simpson \& Kang, 1994; Tabossi \& Laghi, 1992). The present results, however, indicate that it is not only the construction of the list per se that may determine the strategy adopted by the reader. If Korean readers adjusted processing to optimize their average performance over the course of the experiment, we would expect a larger frequency effect any time the list consisted mostly of Hanza words, regardless of any prior information, either in the form of a script cue or the immediately preceding trial(s). Rather, the control of processing is considerably more precise than has previously been assumed.

The results of Experiment 1 show that subjects are influenced by the immediately preceding one or two stimuli. A single Hanza word is sufficient to initiate lexical processing of a subsequent Hangul target, whereas sublexical processing of Hangul requires two preceding Hangul words. We propose that this asymmetry is due to the necessity of lexical processing of Hanza, regardless of context; following a Hanza word there is no question that lexical information has been most recently used. Hangul, on the other hand, can be processed by either path, so following a single Hangul word, the system is not "set" as unambiguously as it is following Hanza. We can only speculate that the naming task is precise enough to detect the shift toward lexical processing that occurs following a Hanza word, but not precise enough for the more subtle, continuous changes in bias toward sublexical processing that arise from prior processing of Hangul words.
In Experiment 2, we showed an even more precise level of strategic control, with subjects being able to control processing on a given trial. This result is consistent with a recent proposal that all aspects of word recognition and reading are under strategic control and are subject to task demands (Balota, Paul, \& Spieler, 2000; Zevin \& Balota, 2000). If characteristics of the experimental context emphasize phonological, orthographic, or semantic information, attention will be focused on that information, and performance will be correspondingly influenced.

It must again be stressed that, although this discussion has relied on the dual-route framework (e.g., Coltheart et al., 1993), a PDP interpretation is equally plausible (e.g., Plaut et al., 1996). As discussed previously, a shallow orthography such as Hangul will tend to show diminished frequency effects (in comparison with those in a deeper orthography) because of the consistency of the direct mapping between orthography and phonology. When the presence of a logographic script directs attention to semantically mediated processing, however, frequency effects for Hangul should be greater. The predictions of such a model for the present experiments, therefore, are essentially the same as those originating in the dualroute account.

Recently, an alternative account of strategic effects in word recognition has been offered. Lupker, Brown, and Colombo (1997) have argued that much of the research on strategy effects can be explained by a mechanism that sets a response deadline in accordance with stimulus characteristics (see also Jared, 1997). That is, they propose that phonological information necessary to articulate a response builds over time, and the subject establishes a criterion, or deadline, for when articulation should begin. The criterion is set according to the average finishing times for stimuli in a block. What appears to be relative emphasis on one type of processing, therefore, may instead be the result of a shift in a response criterion. In addition, in light of the recent studies of Taylor and Lupker (2001) showing that the response deadline is sensitive to the nature of preceding stimuli, it is clear that the finding of local context effects does not automatically compromise a criterion-setting account.

A comparison between the present data and those of Lupker et al. (1997) is not simple because of several important differences in methodology. Nevertheless, we may be able to derive some tentative predictions for the present data from the criterion-setting view. The results of Experiment 1 seem most compatible with this interpretation. The main effect of filler script indicates that the subjects named Hangul words faster in the Hanguldominated than in the Hanza-dominated lists. In addition, because the fillers were selected for their familiarity in Hanza, they tended to be of fairly high frequency themselves and were named quickly. An effect of the overall speeding of responses in the $80 \%$-Hangul lists could be to produce a floor effect on the naming of Hangul words, reducing the difference between high- and low-frequency Hangul word naming. On the other hand, some aspects of Hanza word naming are more difficult to reconcile 
with a criterion-setting account. Hanza naming was not the focus of this research, but a comparison of naming times for 96 Hanza filler words that were common to both Hangul- and Hanza-dominated lists revealed a nonsignificant naming advantage in Hanza-dominated lists $(1,063 \mathrm{msec}$, as opposed to $1,141 \mathrm{msec}$ in the Hanguldominated lists). This result replicates that reported in Simpson and Kang (1994), showing that Hangul, but not Hanza, naming is affected by script proportion. It is not clear why a response deadline would affect the processing of one script and not the other. The finding that words in a particular script are named faster when that script is predominant in the list could be seen as evidence for the setting of a response criterion in accordance with characteristics of the list. However, such a criterion would be set on some basis other than anticipated finishing times for a particular type of word. As stimuli that typically result in slower responses, Hanza words should lead to a higher response deadline and, therefore, to slower naming times for all stimuli, not just Hangul.

The results of Hangul naming in Experiment 2 do not seem compatible with the criterion-setting view. The cuing manipulation is independent of other aspects of the stimuli (e.g., script proportion or preceding trials). An account in criterion-setting terms would appear to require multiple criteria under conscious control of the subjects rather than (or in addition to) adjustments made in response to characteristics of the stimulus list. This seems to be a very different conception of criterion setting from that commonly adopted. The present data, therefore, suggest that there are pathway selection effects in addition to those arising from the setting of a response deadline. What is needed is a more direct comparison across languages of those variables thought to affect a response deadline.

A final question that must be addressed concerns the generality of these results across languages. In what way are the results reported in these studies restricted to shallow orthographies or, indeed, even more specifically, to the two-script system of Korean? As discussed, a number of other studies on shallow orthographies have shown evidence for strategic control over processing (Baluch \& Besner, 1991; Besner \& Hildebrandt, 1987; Simpson \& Kang, 1994; Tabossi \& Laghi, 1992). Similar evidence has been found in English, a much less transparent system (Milota et al., 1997; Monsell et al., 1992). It appears, therefore, that the present results may be quite general. The novel aspect of these data concerns the local control of processing, and it is this characteristic that may be more restricted across languages. It may be that the ability to emphasize one processing route on a trial-by-trial basis rests on the distinction between the routes that is afforded by characteristics of the stimuli. In Korean, the distinction between those stimuli that may be processed easily via sublexical information (Hangul) and those that may not is a dichotomous one. In addition, the visual discrimination between the two types of stimuli is very easy. This allows a certainty over the appropriate route for Korean that is more difficult to achieve in English. In
English, the regularity dimension is a continuous one. That is, some words are more irregular than others (e.g., Cortese, 1998). In addition, regular and irregular words in English are not visually as distinguishable from one another as they are in Hangul and Hanza, since English words are written using the same letters. Finally, in English, it is not the case that a phonological code for pronunciation based on GPC rules cannot be built for irregular wordsit just is not the correct code. For logographic Hanza words, however, it simply is not possible to construct a phonological code from sublexical (i.e., subcharacter) information. Together, these differences suggest that the clarity of the mapping between word type and processing that exists in Korean is not present in English, and it is not unreasonable to think that these differences may be reflected in processing differences between readers of the two languages. With the more continuous nature of spelling-sound mapping in English, we might anticipate greater continuity in the use of lexical and sublexical information in processing English words. In the meantime, the present results indicate that very precise control over the use of lexical and sublexical information is at least possible. It will be for future research to determine whether this degree of flexibility is possible in all languages or is characteristic of only some.

\section{REFERENCES}

Balota, D. A., Paul, S., \& Spieler, D. (2000). Attentional control of lexical processing pathways during word recognition and reading. In S. Garrod \& M. Pickering (Eds.), Language processing (pp. 15-57). London: UCL Press.

BALUCH, B., \& BeSNER, D. (1991). Visual word recognition: Evidence for strategic control of lexical and nonlexical routines in oral reading. Journal of Experimental Psychology: Learning, Memory, \& Cognition, 17, 644-652.

Besner, D., \& Hildebrandt, N. (1987). Orthographic and phonological codes in the oral reading of Japanese Kana. Journal of Experimental Psychology: Learning, Memory, \& Cognition, 13, 335-343.

Cho, J.-R., \& Chen, H.-C. (1999). Orthographic and phonological activation in the semantic processing of Korean Hanja and Hangul. Language \& Cognitive Processes, 14, 481-502.

Clark, H. H. (1973). The language-as-fixed-effect fallacy: A critique of language statistics in psychological research. Journal of Verbal Learning \& Verbal Behavior, 12, 335-359.

Coltheart, M., Curtis, B., Atkins, P., \& Haller, M. (1993). Models of reading aloud: Dual-route and parallel distributed processing approaches. Psychological Review, 100, 589-608.

Cortese, M. J. (1998). Revisiting serial position effects in reading. Journal of Memory \& Language, 39, 652-665.

Frost, R. (1998). Toward a strong phonological theory of visual word recognition: True issues and false trails. Psychological Bulletin, 123, 71-99.

Frost, R., Katz, L., \& Bentin, S. (1987). Strategies for visual word recognition and orthographic depth: A multilingual comparison. Journal of Experimental Psychology: Human Perception \& Performance, 13, 104-115.

JARED, D. (1997). Evidence that strategy effects in word naming reflect changes in output timing rather than changes in processing route. Journal of Experimental Psychology: Learning, Memory, \& Cognition, 23, 1424-1438.

Kang, H., \& Simpson, G. B. (1996). Development of semantic and phonological priming in a shallow orthography. Developmental Psychology, 32, 860-866.

Lee, S. S., Lee, K. D., Nam, K. S., Chung, C. S., Lee, I. K., \& ChoI, 
Y. C. (1991). Development of a corpus of contemporary Korean for compiling a lexicographical database. Seoul: Korean Dictionary Board of Yonsei University.

Lupker, S. J., Brown, P., \& Colombo, L. (1997). Strategic control in a naming task: Changing routes or changing deadlines? Journal of Experimental Psychology: Learning, Memory, \& Cognition, 23, 570-590.

McQuADE, D. V. (1981). Variable reliance on phonological information in visual word recognition. Language \& Speech, 24, 99-109.

Milota, V. C., Widau, A. A., McMickell, M. R., Juola, J. F., \& SimpSON, G. B. (1997). Strategic reliance on phonological mediation in lexical access. Memory \& Cognition, 25, 333-344.

Monsell, S., Patterson, K. E., Graham, A., Hughes, C. H., \& MilROY, R. (1992). Lexical and sublexical translation of spelling to sound: Strategic anticipation of lexical status. Journal of Experimental Psychology: Learning, Memory, \& Cognition, 18, 452-467.

Plaut, D. E., McClelland, J. L., Seidenberg, M. S., \& Patterson, K. E. (1996). Understanding normal and impaired reading: Computational principles in quasi-regular domains. Psychological Review, 103, 56-115.

SCHNEIDER, W. (1988). Micro Experimental Laboratory: An integrated system for IBM PC compatibles. Behavior Research Methods, Instruments, \& Computers, 20, 206-217.

Seidenberg, M. S. (1992). Beyond orthographic depth in reading: Equitable division of labor. In R. Frost \& L. Katz (Eds.), Orthography, phonology, morphology, and meaning (pp. 85-118). Amsterdam: North-Holland.

Shafiullah, M., \& Monsell, S. (1999). The cost of switching between Kanji and Kana while reading Japanese. Language \& Cognitive Processes, 14, 567-607.

Simpson, G. B., \& KANG, H. (1994). The flexible use of phonological information in word recognition in Korean. Journal of Memory \& Language, 33, 319-331.

TABOSSI, P., \& LAGHI, L. (1992). Semantic priming in the pronunciation of words in two writing systems: Italian and English. Memory \& Cognition, 20, 303-313.

TAYLOR, T. E., \& LUPKER, S. J. (2001). Sequential effects in naming: A time-criterion account. Journal of Experimental Psychology: Learning, Memory, \& Cognition, 27, 117-138.

Zevin, J. D., \& Balota, D. A. (2000). Priming and attentional control of lexical and sublexical pathways during naming. Journal of Experimental Psychology: Learning, Memory, \& Cognition, 26, 121-135.

\section{NOTES}

1. No more than three stimuli of the same script ever appeared consecutively. Therefore, it would be possible to predict the script following three consecutive trials of the same script. However, such words were never critical target words, so this predictability should not have influenced the results.

2. In the analyses reported, we included responses to critical Hangul words that were preceded by an error on one of the context trials. This was done simply to maximize the number of trials contributing to the subject and item means. However, additional analyses were conducted that eliminated such trials, with virtually no change in the condition means, and no changes in the patterns of statistical effects.

(Manuscript received October 4, 1999; revision accepted for publication January 16, 2001.) 